Chapter 10

\title{
Role of Autophagy in Burn Wound Progression and Wound Healing
}

\author{
Ligen Li and Mengjing Xiao
}

Additional information is available at the end of the chapter

http://dx.doi.org/10.5772/63711

\begin{abstract}
Background: Burn wound progression refers to the phenomenon of continued tissue loss following abatement of an initial thermal insult, which makes the treatment more difficult, prolongs hospital stay, increases medical costs, and raises the likelihood of scar formation. Autophagy is a highly conserved pathway that delivers intracellular macromolecule waste to lysosomes, where they are degraded into biologically active monomers, such as amino acids, that are subsequently reused to maintain cellular metabolic turnover and homeostasis. We aimed to observe the expression of autophagy in burn wounds in a deep second-degree rodent burn model and further investigate the role of autophagy on burn wound progression and wound healing.
\end{abstract}

Methods: A rat deep second-degree burn model was established by placing a $100^{\circ} \mathrm{C}$ brass rod to the shaved skin of rats for $6 \mathrm{~s}$. Rats were randomly divided into sham burn, burn, burn treated with rapamycin, and burn treated with vehicle. Immediately after creating the thermal injury, rats in the treatment group were given rapamycin $1 \mathrm{mg} / \mathrm{kg}$ by intraperitoneal injection, and the rats in the vehicle group were injected with equivalent carrier solution. Skin tissue specimens from burn wounds were taken for assessment of autophagy, apoptosis, myeloperoxidaseactivity, methanedicarboxylicaldehydecontents, laser Doppler flow values, $\mathrm{H} \& \mathrm{E}$, and Masson staining.

Results: Reduced levels of autophagy, impaired blood flow, together with increased levels of apoptosis, inflammation, and myeloperoxidase activity during the early course of burn wound progression were observed. However, enhanced autophagy in the deep dermal layers was observed, which may function as a prosurvival mechanism against inflammation and ischemia.

Rapamycin enhanced the level of autophagy in burn wounds, ameliorated the early progression of burn depth, and promoted burn wound healing, possibly by protecting the zone of stasis from further necrosis and by reducing the extent or level of apoptosis commonly seen in burn wounds. 
Conclusion: Autophagy protects from burn wound progression and promotes burn wound healing through protecting the zone of stasis from further necrosis, which is probably mediated by phagocytizing damaged organelles to sustain homeostatic response and remodeling in the injured tissue.

Keywords: Autophagy, Burn, injury progression, wound healing, rapamycin

\section{Introduction}

After the initial thermal insult, tissue necrosis is continued in burn wound, which is referred to as the phenomenon of "burn wound progression" [1]. The progression of burn wound is an important problem in clinic, largely determining the morbidity and mortality of burn patients. Generally, the burn wound can be divided into three concentric zones [2]: the zone of coagulation, the zone of stasis, and the zone of hyperemia. While the central zone of coagulation is distinguished by irreversible tissue necrosis and the outer zone of hyperemia can always naturally recover, the intermediate zone of stasis may either progress into necrosis or can be salvaged if optimal treatments be available [3].

It has been demonstrated that burn wound progression is attributed to many mechanisms, including tissue hypoperfusion, prolonged inflammation, edema, hypercoagulability, and free radical damage [4]. Many studies have aimed to ameliorate burn wound progression by intervening these above mechanisms, and certain advance has already been achieved. However, further studies are still needed to help us better understand this complicated process.

Autophagy is a lysosomal-dependent degradation pathway, which is indispensable for survival, development, differentiation, and cellular homeostasis [5]. Autophagy has been reported to protect against ischemic injuries, inflammatory diseases, and other disease models [6]. To understand the role of autophagy in burn wound progression, firstly, we designed an animal study to examine the expressing pattern of autophagy in burn wounds. We found that autophagy level in the zone of stasis was increased when comparing to the normal unburned tissue. We speculated that the increase of autophagy level in the zone of stasis was a survival mechanism against tissue ischemia, excessive inflammatory response, and oxidative stress in burn wounds [7].

Rapamycin is a macrolide antibiotic agent, which was initially used as an antifungal drug, and has potent anti-proliferative and immunosuppressive properties. Rapamycin is now a commonly used autophagy-enhancing agent and it can induce autophagy by inhibiting mTOR kinase activity [8]. In the second part of the work, we used a rat model of second degree burns and selected rapamycin as an autophagic-enhancing agent to determine whether it could enhance autophagy in burn wounds and ameliorate burn wound progression while promoting wound healing. We found that rapamycin enhanced the level of autophagy in burn wounds, ameliorated the early progression of burn depth and promoted burn wound healing, possibly by protecting the zone of stasis from further necrosis and by reducing the extent or level of apoptosis commonly seen in burn wounds [9]. 


\section{Materials and methods}

See details at Refs. [7, 9].

\section{Results}

\subsection{Expression rule of autophagy proteins in burn wounds}

Levels of LC3 and Beclin-1 protein were maintained at a certain degree in normal skin tissue. After burn was inflicted, levels of these proteins declined continuously in wound tissue until $24 \mathrm{~h}$ after the burn. Levels then increased slightly, but remained far lower than in normal skin samples. Quantitative analysis show that autophagy level decreased about fourfold over $24 \mathrm{~h}$, and then began to increase but still could not reach their normal level (Figure 1).

\section{A $\quad$ Con $1 \quad 6 \quad 12 \quad 24 \quad 48 \quad 72(h)$}

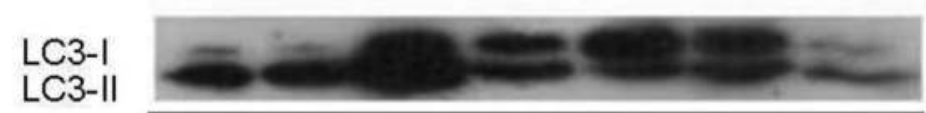

Beclin-1

GADPH
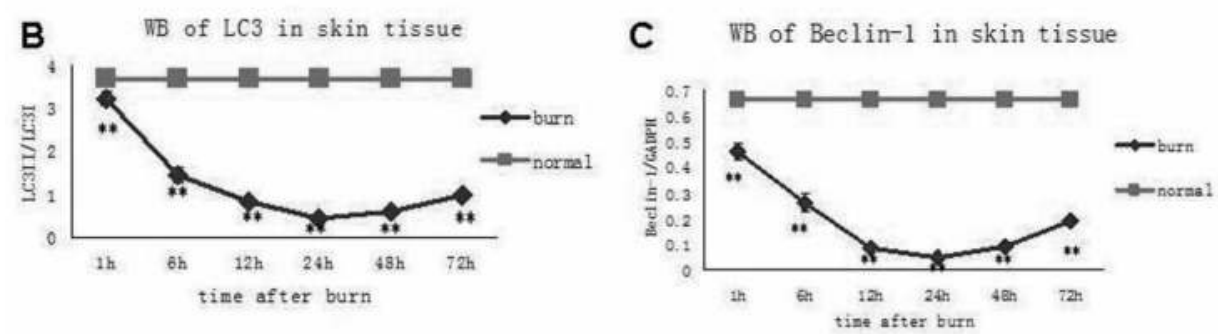

Figure 1. (A) Western blot analyses of LC3 and Beclin-1 in wound and normal unburned skin at different time points after burn. (B, C) Quantitative analysis of LC3II/I and Beclin-1 in burn wounds. B and C show that the levels of these proteins declined continuously in wound tissue until $24 \mathrm{~h}$ after the burn. Levels then increased slightly, but remained far lower than in skin samples. The times trends for LC3II/I and Beclin-1 change were almost identical. Data are presented as means $\pm \mathrm{SD}\left({ }^{*} P<0.05,{ }^{* *} \mathrm{P}<0.01, \mathrm{n}=8\right.$ per group).

\subsection{Change of apoptosis level in burn wounds}

To investigate the apoptosis level of burn wounds, we conducted TUNEL staining to see how the apoptosis level in burn wounds would change after burn was inflicted. We found more 
cells undergoing apoptosis in burn wounds than control. The apoptotic rates in burn wounds at $6,24,48$, and $72 \mathrm{~h}$ are $4.56 \pm 0.27,11.76 \pm 0.7,13.16 \pm 0.65$, and $8.14 \pm 0.62 \%$, respectively, versus $2.23 \pm 0.23 \%$ of controls (mean $\pm \mathrm{SD}, P<0.05$ at $6 \mathrm{~h}, P<0.01$ at $24,48,72 \mathrm{~h}$ ) (Figure 2).

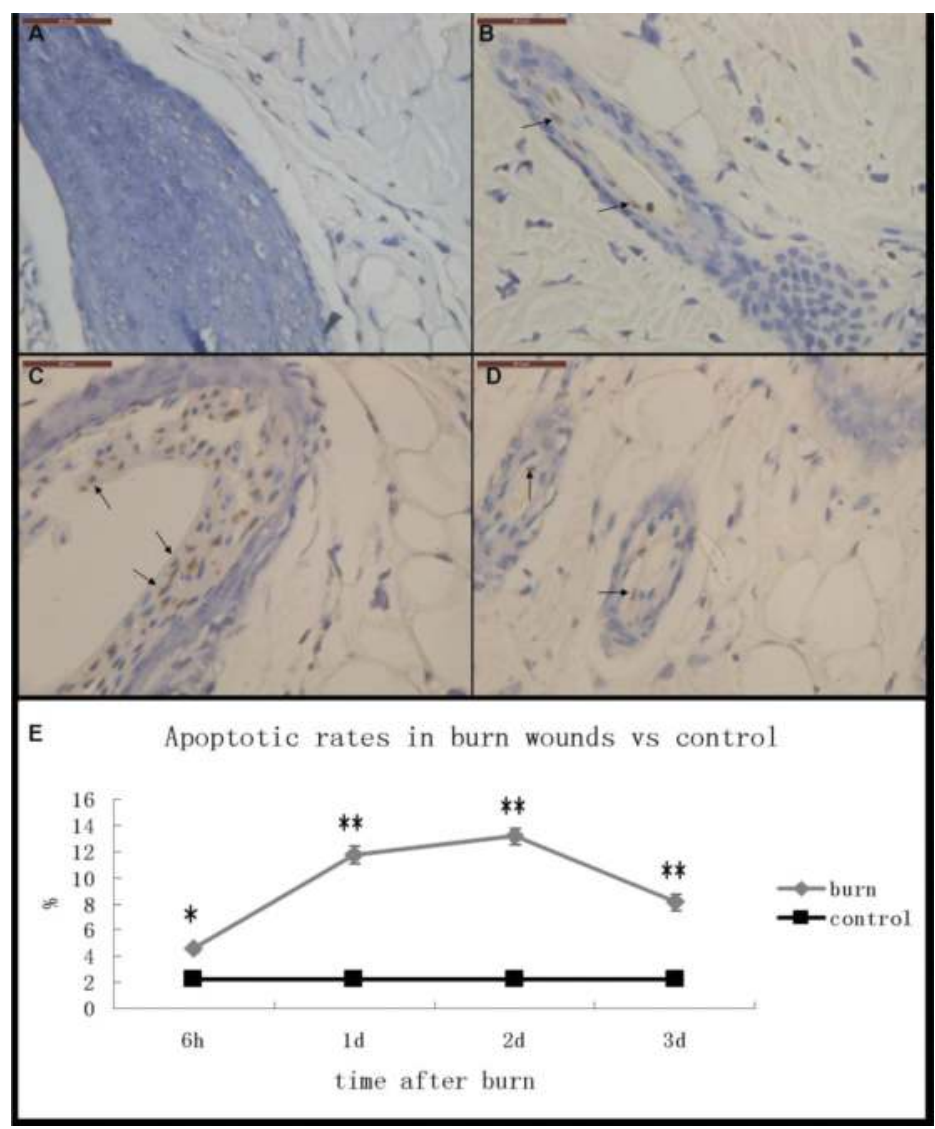

Figure 2. TUNEL staining of normal skin (A) and burn wound tissue 1 day (B), 2 days (C), and 3 days (D) post burn. Cells with shrunken brown stained nuclei were considered positive (black arrows). E: The $p$ values for $6 \mathrm{~h}, 1 \mathrm{~d}, 2 \mathrm{~d}$ and $3 \mathrm{~d}$ are $0.028,0.005,0.003$ and 0.007 respectively. Scale bars $=50 \mathrm{um},{ }^{*} P<0.05,{ }^{* *} P<0.01, \mathrm{n}=8$ per group.

\subsection{Change of Laser Doppler Flowmetry (LDF) value and Myeloperoxidase (MPO) activity in burn wounds}

LDF value in burn wounds is an indicator of burn wound blood flow, while MPO activity reflects the inflammatory response in the burn wound. We found that the LDF values decreased fourfold over $12 \mathrm{~h}$ after burn and then began to increase but still far lower normal level. The MPO activity increased about 5.3-fold over $48 \mathrm{~h}$ after burn and then decreased slightly, yet still significantly higher than control (Figure 3). 


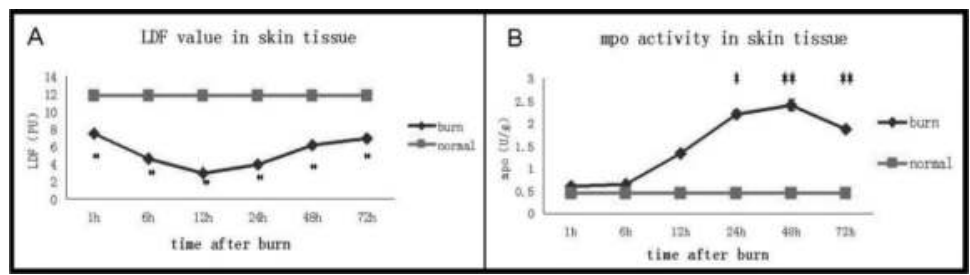

Figure 3. LDF value and MPO activity in burn wounds and normal skin. (A) LDF values in the burn wounds were significantly decreased as compared to the control group. LDF values in the burn wounds decreased fourfold over $12 \mathrm{~h}$ and then began to rise, yet still could not reach their normal level. (B) MPO activities in burn wounds were significantly increased than the control. The levels of MPO activity in burn wounds were increased 5.3-fold over $48 \mathrm{~h}$, and then decreased but still far higher than normal. Alterations in the LDF value were significant at all the time points post burn. Changes in the levels of MPO activity between burn and control groups were significant at the time points of 1 , 2 , and 3 days post burn. The values described herein were mean \pm SD (burn versus control group, ${ }^{*} P<0.05,{ }^{* *} P<0.01, \mathrm{n}$ $=8$ per group).

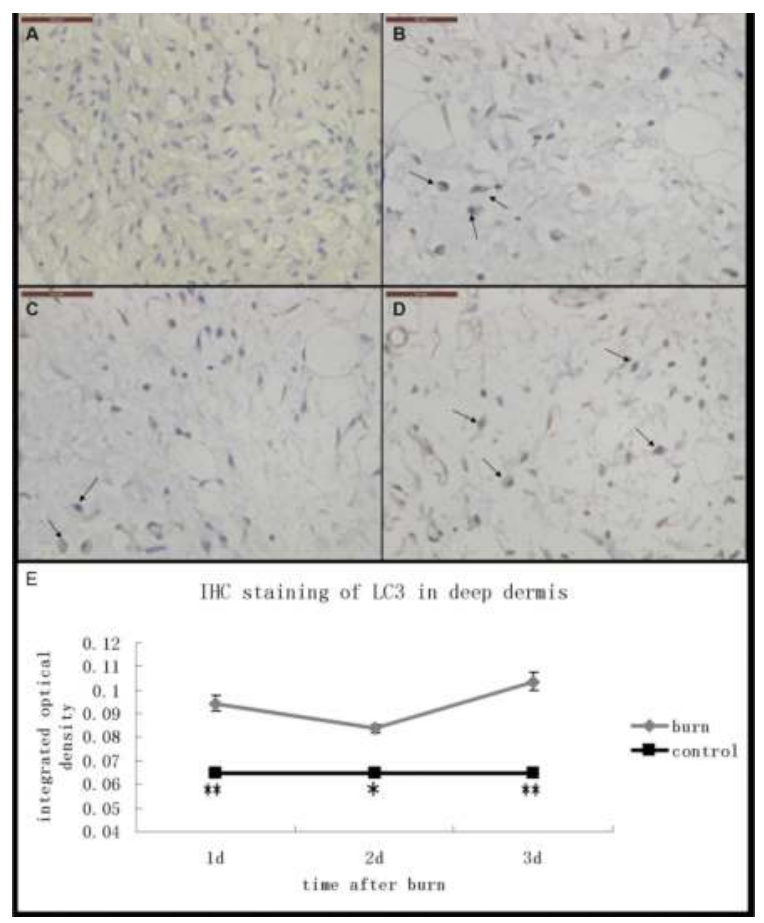

Figure 4. Immunohistochemical staining of LC3 (black arrows, cells with brown cytoplasm) in deep dermis of normal skin (A) and burn wounds (B-D). (B) 1 day post burn; (C) 2 days post burn; (D) 3 days post burn. At all time points after burn, the cells expressing LC3 were increased in the deep dermis of burn wound tissue as compared to that of control. Scale bars $=50 \mathrm{um}$. (E) Semi-quantitative analysis of the immunohistochemical staining results. The expression of LC3 in the deep dermis of burn wounds was significantly higher than that of the control at all time points. The values herein were mean $\pm \mathrm{SD}\left({ }^{*} P<0.05,{ }^{* *} P<0.01, \mathrm{n}=8\right.$ per group). 


\subsection{LC3 staining in the zone of stasis}

We found that LC3-positive cells in the deep dermis adjacent to the subcutaneous tissue, which in this deep second-degree burn model, may represent the zone of stasis, which were much more than that of control. We conducted a quantitative analysis and found that the increase of LC3-positive cells in the deep dermis was significant at 6, 24, and $48 \mathrm{~h}$ post burn (Figure 4).

\subsection{Rapamycin enhanced the autophagic level in burn wounds}

Western bolt analysis showed that the protein levels of two autophagy markers, LC3 and Beclin-1, were significantly augmented in the thermal burn wounds of the rapamycin-treated group as compared with the levels seen in the control group (Figure 5). The expression of both proteins in burn wounds were at their lowest $24 \mathrm{~h}$ post-thermal burn injury and then gradually increased with time. The patterns of LC3-II/LC3-I and Beclin-1 expression were almost identical (Figure 5). Moreover, the increases in expression of these proteins were statistically significant at $6 \mathrm{~h}(p<0.05)$ and at 24 and $48 \mathrm{~h}$ post-thermal burn injury $(p<0.01)$.
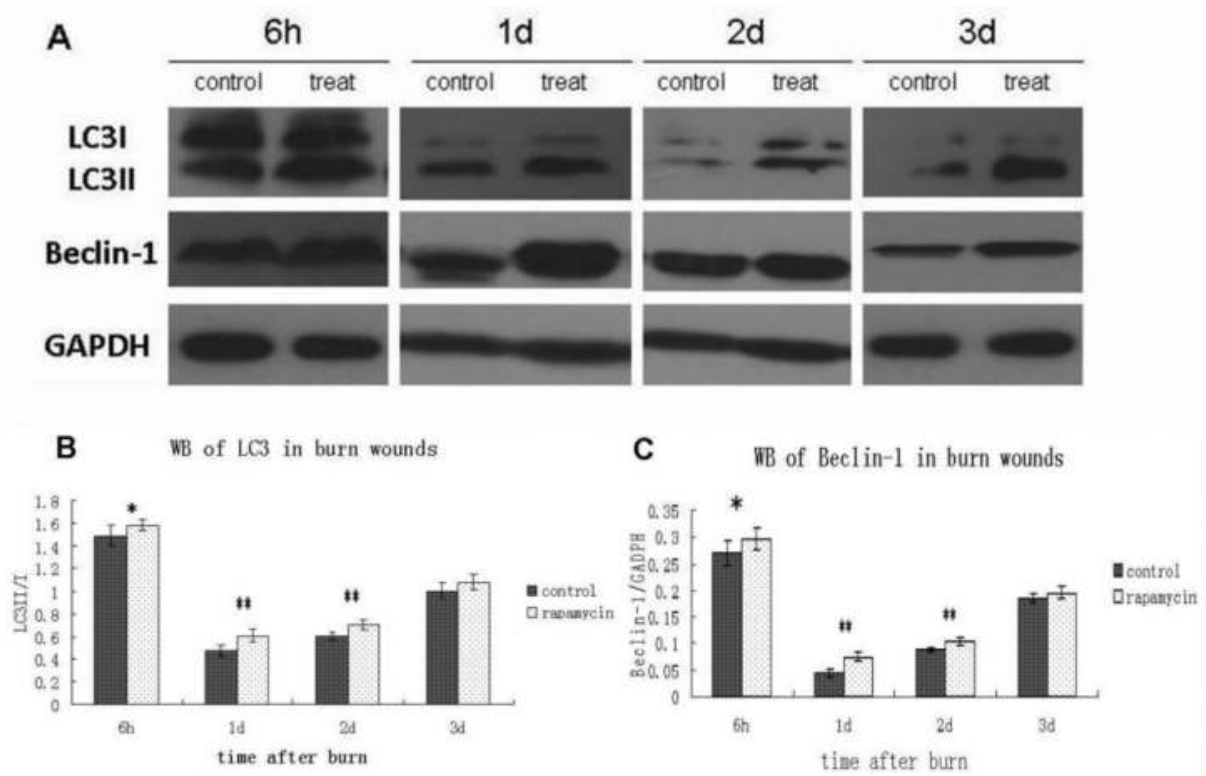

Figure 5. Western immunoblot assay of LC3 and Beclin-1 expression in burn wounds of control and treatment groups (A-C). The values herein were mean $\pm \mathrm{SD}\left({ }^{*} p<0.05,{ }^{* *} p<0.01, \mathrm{n}=8\right.$ per group).

\subsection{Rapamycin treatment inhibited apoptosis in burn wounds}

To further investigate if the enhanced autophagy had any effects on apoptosis of burn wounds, we conducted TUNEL staining to see if the apoptosis level in burn wounds of treated rats were different from controls. Cells with shrunken brown stained nuclei were considered positive. 
We found fewer cells undergoing apoptosis in rats treated with rapamycin. The apoptotic rates in burn wounds of treated rats at $6,24,48$, and $72 \mathrm{~h}$ are $2.99 \pm 0.33,8.85 \pm 0.6,12.48 \pm 0.67$, and $6.7 \pm 0.31 \%$, respectively, versus $4.56 \pm 0.27,11.76 \pm 0.7,13.16 \pm 0.65$, and $8.14 \pm 0.62 \%$ of controls (mean \pm SD , $p<0.05$ at 6, 24, $72 \mathrm{~h}$ ). Besides, we found that the TUNEL-positive cells were mainly observed in the epithelium of hair follicles or blood vessels (Figure 6).

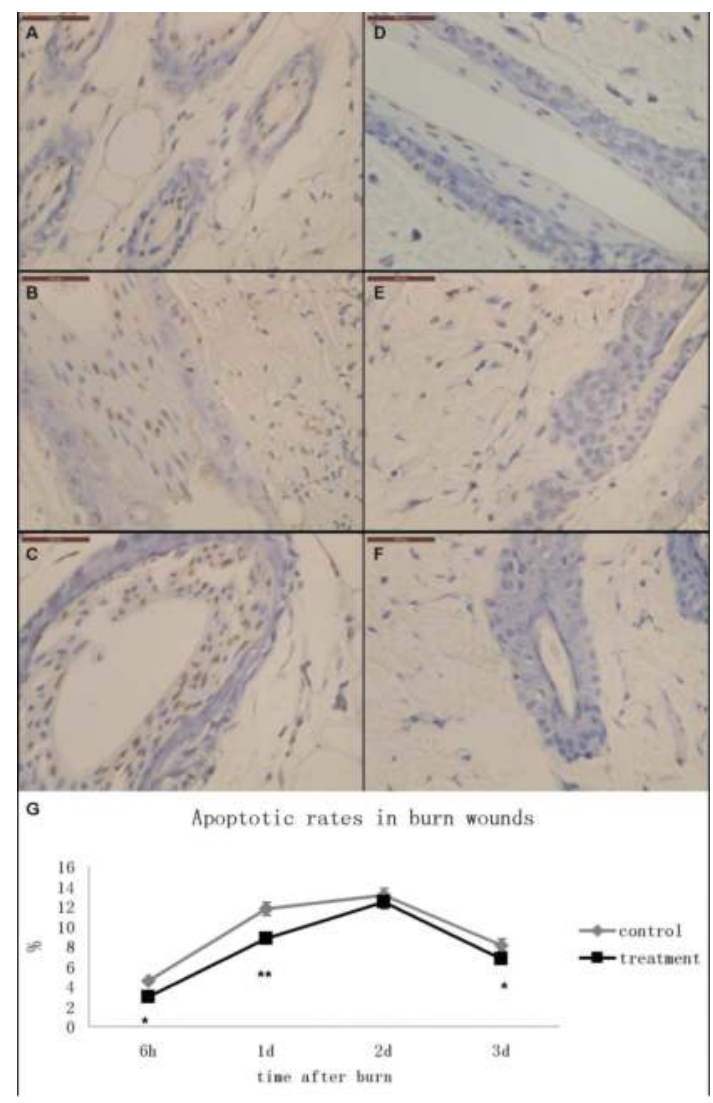

Figure 6. TUNEL staining of burn wounds in control (A-C) and treatment (D-F). (A, D) $6 \mathrm{~h}$ post burn; (B, E) $24 \mathrm{~h}$ post burn; (C, F) $72 \mathrm{~h}$ post burn. TUNEL positive cells were characterized by shrunken nucleus with brown staining. Scale bars $=50 \mathrm{um}$. (G) Quantitative analyses of TUNEL staining results. The values herein were mean $\pm \mathrm{SD}\left({ }^{*} p<0.05,{ }^{* *} p<\right.$ $0.01, \mathrm{n}=6$ per group).

\subsection{Rapamycin treatment reduced inflammatory infiltration and restored blood perfusion in burn wounds}

The LDF values and $\mathrm{Na} / \mathrm{K}$-ATPase activities in burn wounds of the treatment group were significantly increased when compared to those of the control group (Figure 7A, B). Furthermore, both LDF values and $\mathrm{Na} / \mathrm{K}$-ATPase activities in burn wounds were lower than those of 
normal unburned skin. The IL-8 level, MPO activity, and MDA content in burn wounds of the treatment group were significantly decreased when compared to those of the control group. In addition, these measurements were almost all higher than those of normal unburned skin (Figure 7C-E). Changes in the activities of $\mathrm{Na} / \mathrm{K}-\mathrm{ATPase}$ and MPO between the treatment and control groups were significantly different at the time points of 1 day and 2 days post-thermal burn injury. The changes in LDF, MDA, and IL- 8 content between the treatment and control groups were also significant at the time points of 1,2 , and 3 days post-thermal burn injury.
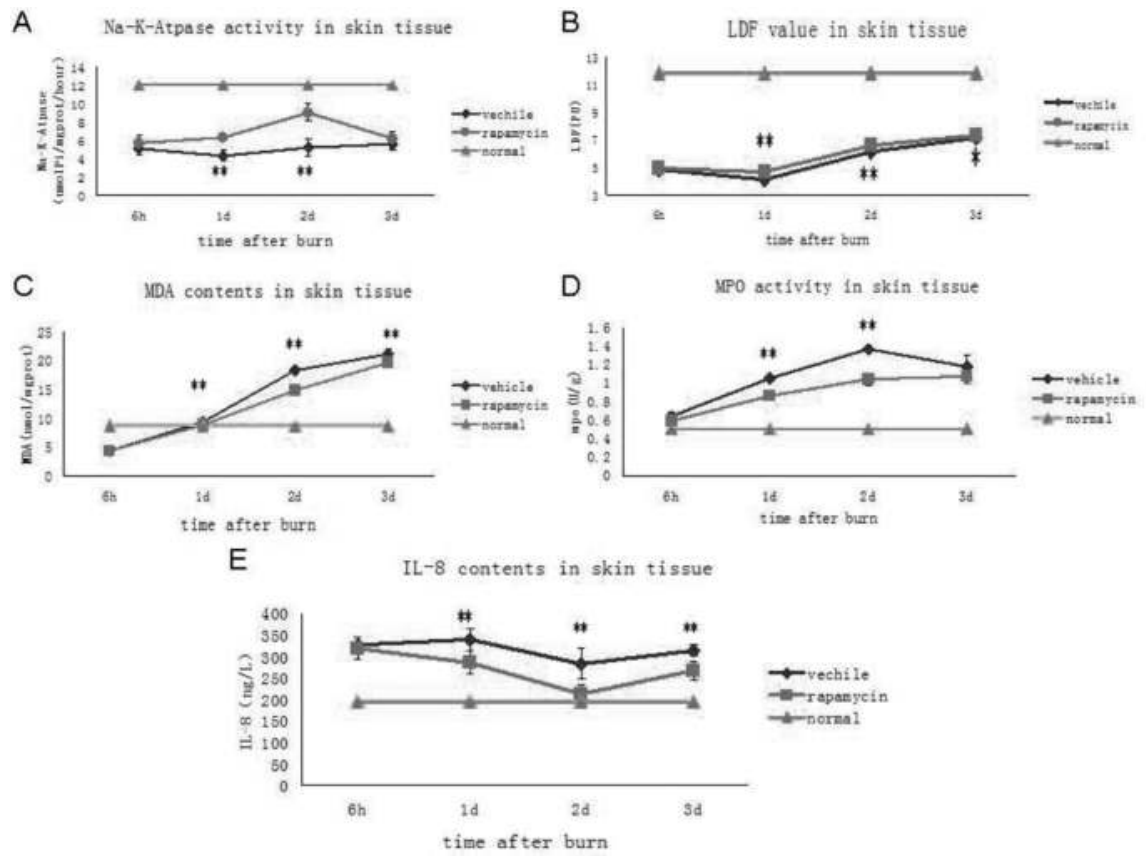

Figure 7. Na/K-ATPase activities, LDF values, MDA contents, MPO activities, and IL- 8 contents in burn wounds of the treatment group, vehicle control, and normal unburned control. A: The $p$ values for $1 \mathrm{~d}$ and $2 \mathrm{~d}$ are 0.008 and 0.006 . B: The $p$ values for $1 \mathrm{~d}, 2 \mathrm{~d}$ and $3 \mathrm{~d}$ are $0.007,0.007$ and 0.043 , respectively. C: The $p$ values for $1 \mathrm{~d}, 2 \mathrm{~d}$ and $3 \mathrm{~d}$ are $0.009,0.006$ and 0.008 , respectively. D: The $p$ values for $1 \mathrm{~d}$ and $2 \mathrm{~d}$ are 0.005 and 0.003 . E: The $p$ values for $1 \mathrm{~d}, 2 \mathrm{~d}$ and $3 \mathrm{~d}$ are 0.006 , 0.004 and 0.006 , respectively. The values described herein were mean \pm SD (treatment versus control group, ${ }^{*} p<0.05$, ${ }^{* *} p<0.01, \mathrm{n}=8$ per group).

\subsection{Rapamycin treatment reduced burn wound progression}

Histopathological examination demonstrated that the burn wound progression was reduced in the treatment group when compared to the controls (Figure 8). Fewer residual hair follicles, 
deeper tissue necrosis, more severe collagen denaturation, and more inflammatory infiltrations in burn wounds of the control group were seen when compared to those of the treatment group. Masson staining showed that less intense staining with Masson red were seen in the treated rats when compared to control, which may indicate less tissue necrosis in the treated rats (Figure 8A-F).

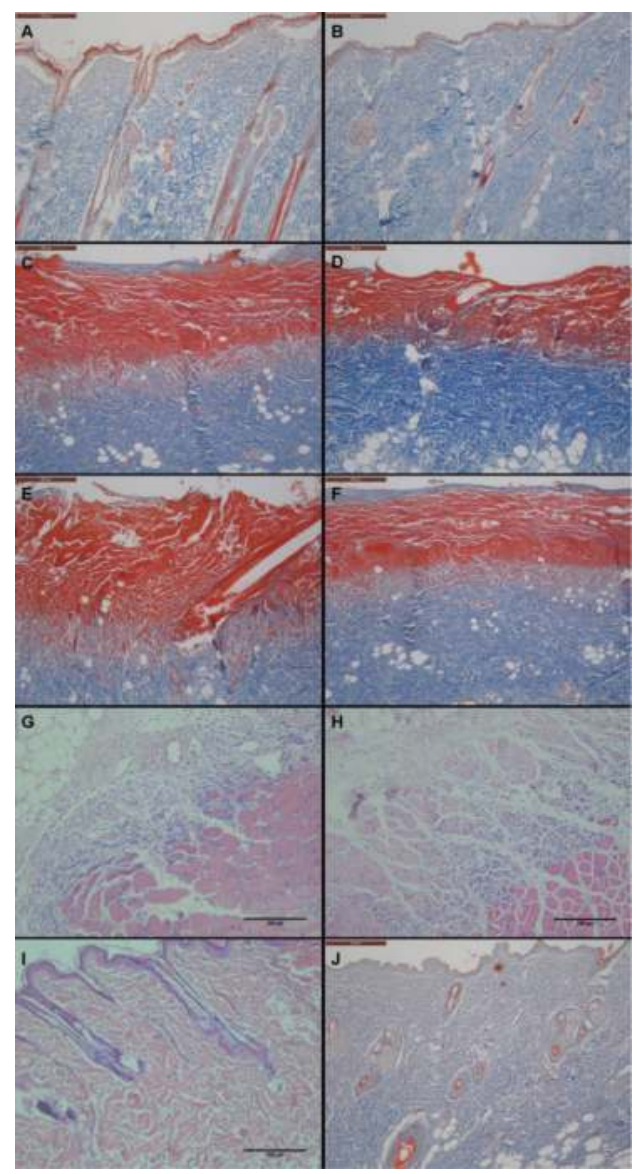

Figure 8. H\&E and Masson staining of normal skin tissue and burn wounds. (A-F) Masson staining of burn wounds of 1-day control (A), 1 day following treatment (B), burn wounds of 2-day control (C), 2 days following treatment (D), 3day control $(E)$, and 3 days following treatment $(F) .(G, H) H \& E$ staining of 3-day control $(G)$, and 3 days following treatment $(\mathrm{H})$. (I, J) H\&E and Masson staining of normal skin tissue. Scale bars $=200 \mathrm{um}$.

\subsection{Rapamycin treatment promoted burn wound healing}

Finally, the time to wound re-epithelialization was shorter in treated rats as compared with controls ( $22.5 \pm 1.4$ vs. $24.8 \pm 1.3$ days, respectively). (mean \pm SD, $p<0.01$, Figure 9). 
Time to wound reepithelialization of burned rats

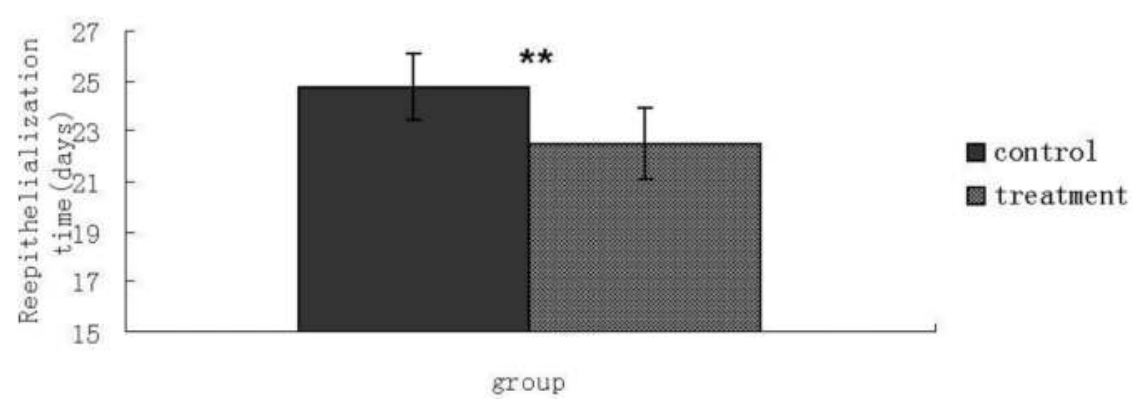

Figure 9. Time to wound re-epithelialization. The time to wound re-epithelialization was significantly shorter in treated rats compared with controls.

\section{Discussion}

The phenomenon of burn wound progression has long been considered a complex and challenging clinical and theoretical challenge. For over half a century, investigators in the field have been attempting to study the mechanisms of burn wound penetration of the epidermal and dermal layers of the skin with the objective of gaining greater insights into the pathology. Several studies [10] have demonstrated that a number of factors contribute to the invasive deepening of thermal burn wounds including a deficiency in local burn wound perfusion, edema, thrombosis, free radical damage, accumulation of factors that contribute to cellmediated toxicity and inflammatory cell infiltration.

The first part of our study showed that after the occurrence of burn injury, laser Doppler flow values and $\mathrm{Na} / \mathrm{K}$-ATPase enzyme activities, which collectively represent the burn wound blood circulation and index of energy metabolism, decreased significantly. Moreover, both MPO and MDA activities, which respectively represent wound inflammation and free radical generation, increased significantly. Moreover, the autophagy level was at first reduced and then increased, yet remained far below those in normal skin. We speculate that at the early stage when the tissue necrosis due to thermal injury was inevitable, autophagy may not be a principal cellular activity as necrosis did in burn wounds and which is why autophagy level decreased at first. At the latter stage, when the inevitable tissue necrosis ceased, the remain viable tissue adjacent to coagulation was subject to ischemic and inflammatory damage among others, and then autophagy may protect against these stress stimuli as a survival mechanism which account for the latter rise of autophagy level. The prosurvival role of autophagy is supported by our another finding in IHC staining, which demonstrated significantly activated autophagy in the deep dermis which in this burn model, and represents the zone of stasis (or ischemia). In addition, at the latter stage (48-72 h) after burn, tissue perfusion as determined by LDF was restored and inflammatory infiltration in burn wounds as shown by MPO activity 
was reduced, in coincidence with the increase of autophagy level at the same time, which may add to evidence for the pro-survival roles of autophagy in this burn model.

To further dissect the role of autophagy in burn wound progression, we used rapamycin as treatment intervention in the second part of the study. The results demonstrated that the autophagic levels in burn wounds were enhanced following treatment with rapamycin. Furthermore, both LDF values and $\mathrm{Na} / \mathrm{K}$-ATPase activities (indicators of blood perfusion and energy metabolism) in the wounds of treatment group were significantly increased as compared to those of the control group. However, the IL-8 level, MPO activities, and MDA content (representative of inflammatory reaction and free radial damage) in the wounds of rapamycintreated group were significantly decreased as compared to those of the control group [11]. Therefore, these results suggested that enhanced autophagy restored blood perfusion and energy metabolism, and inhibited inflammatory reaction and oxidative damage in burn wounds. Thus, it can be inferred that autophagy maintained cellular metabolic turnover and homeostasis in this experimental burn model [12].

Histopathological results showed that the burn wound depth of the rapamycin-treated group was less remarkable and "more superficial" than that seen in the control group, particularly at 2 and 3 days following thermal tissue injury. Additionally, as compared with the burn wounds of the treatment group, there were fewer residual hair follicles, more severe denatured collagen events recorded, evidence of deeper tissue necrosis, and more obvious inflammatory infiltrates in the burn wounds of the control group. These results suggested that burn wound progression in the treated rats was ameliorated. At last, burn wounds in treatment group reepithelialized faster than those of control group, further indicating that prevention of burn wound progression were beneficial to burn wound healing.

\section{Conclusion}

Autophagy protects from burn wound progression and promotes burn wound healing through protecting the zone of stasis from further necrosis, which is probably mediated by phagocytizing damaged organelles to sustain homeostatic response and remodeling in the injured tissue.

\section{Author details}

Ligen $\mathrm{Li}^{*}$ and Mengjing Xiao

*Address all correspondence to: liligen@126.com

The Department of Burn and Plastic Surgery, Burn Institute, First Affiliated Hospital of General Hospital of PLA, Beijing, China 


\section{References}

[1] Shupp JW, Nasabzadeh TJ, Rosenthal DS, Jordan MH, Fidler P, Jeng JC. A review of the local pathophysiologic bases of burn wound progression. J Burn Care Res. 2010;31:84973.

[2] Jackson DM. [The diagnosis of the depth of burning]. Br J Surg. 1953;40:588-96.

[3] Jackson DM. Second thoughts on the burn wound. J Trauma. 1969;9:839-62.

[4] Singh V, Devgan L, Bhat S, Milner SM. The pathogenesis of burn wound conversion. Ann Plast Surg. 2007;59:109-15.

[5] Levine B, Kroemer G. Autophagy in the pathogenesis of disease. Cell. 2008;132:27-42.

[6] Deretic V, Saitoh T, Akira S. Autophagy in infection, inflammation and immunity. Nat Rev Immunol. 2013;13:722-37.

[7] Xiao M, Li L, Li C, et al. Role of autophagy and apoptosis in wound tissue of deep second-degree burn in rats. Acad Emerg Med. 2014;21:383-91.

[8] Vignot S, Faivre S, Aguirre D, Raymond E. mTOR-targeted therapy of cancer with rapamycin derivatives. Ann Oncol. 2005;16:525-37.

[9] Xiao M, Li L, Hu Q, et al. Rapamycin reduces burn wound progression by enhancing autophagy in deep second-degree burn in rats. Wound Repair Regen. 2013;21:852-9.

[10] Tobalem M, Harder Y, Schuster T, Rezaeian F, Wettstein R. Erythropoietin in the prevention of experimental burn progression. Br J Surg. 2012;99:1295-303.

[11] Schmauss D, Rezaeian F, Finck T, Machens HG, Wettstein R, Harder Y. Treatment of secondary burn wound progression in contact burns - a systematic review of experimental approaches. J Burn Care Res. 2015;36:e176-89.

[12] Levine B, Mizushima N, Virgin HW. Autophagy in immunity and inflammation. Nature. 2011;469:323-35. 